\title{
A PANDEMIA POR COVID-19 E O PACIENTE IDOSO: QUAIS AS NECESSIDADES DE ATENÇÃO EM SAÚDE PARA ESSE GRUPO POPULACIONAL'?
}

\author{
THE COVID19 PANDEMIC AND THE ELDERLY PATIENT: \\ WHAT ARE THE HEALTH CARE NEEDS FOR THIS POPULATION GROUP?
}

\author{
Laura Goulart ${ }^{2}$, Maíra de Oliveira Penna ${ }^{3}$, Fernanda Real Dotto ${ }^{4}$, \\ Tereza Cristina Blasi $^{5}$ e Jane Beatriz Limberger ${ }^{6}$
}

\begin{abstract}
RESUMO
Objetivo: Este artigo visa abordar as diferentes facetas da vulnerabilidade do paciente idoso durante a pandemia por COVID-19, com foco nos impactos biológicos, psicológicos e sociais da doença. Metodologia: Foi realizada uma pesquisa bibliográfica utilizando os descritores "SARSCov-2" OU "COVID-19" E "ELDERLY PATIENT" e os resultados foram agrupados de acordo com as temáticas apresentadas nos artigos. Resultados: A análise da literatura publicada sobre a pandemia demonstra que as políticas de isolamento social dos idosos precisam estar alinhadas às ações de saúde e assistência social para dar conta da diversidade de necessidades destes pacientes. Conclusão: É necessário, portanto, manter o foco na prevenção da COVID-19 aliada à outras demandas preventivas da saúde física e emocional dos idosos durante pandemias.
\end{abstract}

Palavras-chave: COVID-19, Idoso, Geriatria, Pandemia, Vulnerabilidade.

\section{ABSTRACT}

Objective: This article aims to address the different facets of the vulnerability of this age group during the COVID-19 pandemic, focusing on the biological, psychological and social impacts of the disease. Methodology: A bibliographic search was performed using the descriptors "SARSCov-2" OR "COVID-19" AND "ELDERLY PATIENT" and the results were grouped according to the themes presented in the articles. Results: Analysis of the published literature on the pandemic demonstrates that policies of social isolation for the elderly need to be aligned with health and social assistance actions to account for the diversity of needs of these patients. Conclusion: It is therefore necessary to maintain the focus on the prevention of COVID-19 combined with other preventive demands on the physical and emotional health of the elderly during pandemics.

Keywords: COVID-19, Elderly, Geriatric Medicine, Pandemic, Vulnerability.

\footnotetext{
${ }^{1}$ Revisão Bibliográfica - PROBEX-UFN

${ }^{2}$ Autora. Acadêmcia do curso de Psicologia - Universidade Franciscana. laurasma@hotmail.com

${ }^{3}$ Colaboradora. Acadêmica do curso de Nutrição - Universidade Franciscana. maira.penna@ufn. edu.br

${ }^{4}$ Colaboradora. Docente do curso de Psicologia - Universidade Franciscana. fernandareal@hotmail.com

${ }^{5}$ Colaboradora. Docente do curso de Nutrição - Universidade Franciscana. tcb29@hotmail.com

${ }^{6}$ Orientadora. Docente do curso de Farmácia - Universidade Franciscana. janebeatriz@ufn. edu.br
} 


\section{INTRODUÇÃO}

A pandemia COVID-19 afetou mais de 180 países e 5 milhões de pessoas em todo o mundo nos primeiros seis meses de disseminação da doença. Enquanto o mundo procura se situar e produzir pesquisa sobre a rapidez da propagação e taxas de infecção e morbimortalidade, torna-se necessário voltar nosso olhar para a população que vem sendo mais atingida pela doença - os idosos. Observa-se uma diferença entre as características da infecção em cada um dos países atingidos, como a epidemiologia, vias de infecção, apresentação clínica e protocolos de tratamento, mas o fato de que o idoso é atingido com maior gravidade é observado como principal característica dessa doença (TRIPATHY, 2020).

De maneira geral, tanto o isolamento social quanto a solidão estão associados ao aumento da mortalidade na população idosa, mas é incerto se seus efeitos são independentes ou se a solidão representa o caminho emocional pelo qual o isolamento social prejudica a saúde (STEPTOE, 2013). O isolamento social entre os idosos é um sério problema de saúde pública devido ao risco elevado de problemas cardiovasculares, autoimunes, neurocognitivos e de saúde mental. Desta forma torna-se necessário o estabelecimento de políticas públicas que garantam o suprimento de mantimentos e medicamentos vitais para evitar a progressão de comorbidades. Além disso, o contato com a família ou amigos próximos, além dos serviços de apoio voluntários e de assistência social desempenham um importante papel para os idosos sozinhos, isolados ou reclusos (ARMITAGE; NELLUMS, 2020).

Em função da temática recente, observa-se a incipiência de estudos, em especial os de revisão, que abarquem a temática da pandemia do COVID-19 e seu impacto na população idosa. Nesse contexto, este artigo visa verificar o impacto da pandemia por COVID-19 na saúde da população idosa, bem como verificar estratégias de cuidado baseadas nas necessidades deste grupo populacional em função do isolamento e do risco de morbimortalidade.

\section{MATERIAL E MÉTODOS}

Foi realizada uma pesquisa bibliográfica nas bases de dados PUBMED, SCIENCE DIRECT e GOOGLE SCHOLAR utilizando os descritores "SARSCov-2" OU "COVID-19" E "ELDERLY PATIENT". A pesquisa ocorreu nos meses de julho e agosto de 2020, priorizando ensaios clínicos, estudos de revisão, estudos observacionais e guidelines oficiais. Foram buscados artigos no idioma inglês, preferencialmente publicados no ano de 2020. A partir dos artigos encontrados, foram levantadas as seguintes temáticas que serviram de base para a discussão: 1) Manifestações clínicas da COVID19 em idosos; 2) Problemas de saúde mental em idosos em função da pandemia; 3) Aspectos éticos e sociais relacionados ao cuidado e alocação de recursos; 4) Prevenção, reabilitação e paliação de COVID-19 em idosos; 5) Higiene alimentar e segurança nutricional; e 6) Pesquisas para o tratamento farmacológico da COVID-19 em idosos. 


\section{RESULTADOS E DISCUSSÃO}

\section{MANIFESTAÇÕES CLÍNICAS DA COVID-19 EM IDOSOS}

O sistema imunológico dos idosos sofre inúmeras alterações relacionadas à idade, denominadas coletivamente senescência imunológica. Essas mudanças afetam muitos elementos celulares e moleculares tanto do sistema imunológico inato quanto do adaptativo, bem como a coordenação da própria resposta no tempo e no espaço, que funciona de forma eficaz em indivíduos jovens e adultos, mas se deteriora com a idade. A soma dessas mudanças deixa os idosos particularmente vulneráveis a doenças infecciosas, como é o caso da COVID-19 (BUHEJI, 2020).

Teorias apontam que pode haver uma associação entre os níveis da enzima conversora de angiotensina (ACE) 2 e infecção por COVID-19. Pacientes idosos com comorbidades como obesidade e diabetes podem apresentar níveis aumentados de ACE2, elevando à suscetibilidade e à gravidade da infecção por COVID-19. Embora os efeitos a longo prazo da infecção por COVID-19 em humanos não sejam claros, o efeito potencial do excesso de citocinas inflamatórias pode elevar a morbimortalidade pela doença em função da possível deterioração cognitiva e desencadeamento de doenças cardiovasculares (OMURA et al., 2020).

Outra hipótese para o aumento da gravidade da doença por COVID-19 em idosos está relacionada ao surgimento da "tempestade de citocinas", uma rápida e descontrolada cascata de sinalização inflamatória, que exacerba a dispneia e a hipoxemia e desencadeia inflamação nos principais tecidos, como pulmões, rins, coração, fígado e cérebro. A inflamação vascular resultante está emergindo como a principal causa de lesão microvascular associada ao complemento e trombose em casos graves da doença. No idoso, os níveis de dímero D, o principal prognóstico da coagulopatia, aumenta naturalmente com a idade, portanto, o teste de dímero D tem uma alta taxa de falsos positivos em idosos, refletindo um nível mais alto de inflamação vascular. Em tempestades de citocinas, altos níveis de IL-6 induzem a cascata da coagulação, levando a episódios tromboembólicos que elevam a mortalidade pela doença nesta faixa etária (MUELLER; MCNAMARA; SINCLAIR, 2020).

Estudos têm relacionado a microbiota intestinal como fator protetivo para a COVID-19, e supõe-se que a microbiota intestinal regula significativamente o desenvolvimento e a função do sistema imunológico inato e adaptativo. Os pacientes idosos e imunocomprometidos têm uma microbiota intestinal menos diversa e microrganismos benéficos - bifideobactéria, perdem terreno. Ao contornar uma resposta a infecções patogênicas como o coronavírus, um microbioma intestinal saudável pode ser fundamental na manutenção de um sistema imunológico ideal para prevenir uma série de reações imunológicas excessivas que eventualmente se tornam prejudiciais aos pulmões e sistemas de órgãos vitais. Em tais circunstâncias, torna-se imperativo ter uma resposta imunológica balanceada, em que uma resposta superreativa ou subreativa pode igualmente agravar complicações clínicas (DHAR; MOHANTY, 2020). 
Como vimos, diversas teorias têm sido levantadas para explicar a história natural da doença em indivíduos idosos, elevando os riscos de morbimortalidade nesta faixa etária. Contudo, mais pesquisas são necessárias para investigar os efeitos do COVID-19 em várias doenças relacionadas à idade, que possam aumentar a fragilidade do idoso e o risco para sua saúde.

\section{PROBLEMAS DE SAÚDE MENTAL EM IDOSOS EM FUNÇÃO DA PANDEMIA}

O isolamento social imposto pela pandemia por COVID-19 coloca o idoso em uma categoria de elevado risco para outros problemas de saúde física e mental. São descritos casos de exacerbação e recidivas de quadros existentes de medos, fobias, transtornos de ansiedade e transtorno obsessivo-compulsivo, que podem evoluir para transtorno de estresse pós-traumático (GIRDHAR; SRIVASTAVA; SETHI, 2020).

A magnitude da pandemia permite a realização de ensaios controlados randomizados destinados a examinar os efeitos do comportamento cognitivo com base em terapias administradas de maneira remota ou pessoalmente, demonstrando capacidade de aliviar os sintomas de depressão e ansiedade enquanto diminui a sensação de solidão (NEWMAN; NUR, 2020).

O manejo destes transtornos pode ser viabilizado por meio de medidas tais como intervenções que promovam a interação social, terapias psicológicas, prestação de cuidados de saúde e incentivo às atividades de lazer, podendo ser úteis na mitigação das consequências para a saúde mental durante o isolamento. Além disso, a promoção da sensação de segurança física e social, da esperança, da conexão, da calma e da consciência de comunidade são eficazes no controle dos efeitos do isolamento social. Essas ações são viáveis inclusive via remota por meio de plataformas de interação social mediadas pela internet (KÄLL et al, 2020; GIRDHAR; SRIVASTAVA; SETHI, 2020).

Ainda não é possível inferir se a pandemia por COVID-19 influencia as taxas de suicídio em idosos, no entanto, é provável que a pandemia resulte em uma confluência dos fatores de risco para comportamentos suicidas. No entanto, existem elementos para a prevenção do suicídio em idosos que podem reduzir esses indicadores, tais como a disseminação acessível de informações precisas, promovendo autoajuda e enfrentamento positivo, reduzindo isolamento por meio da tecnologia e desenvolvimento do Telessaúde (WAND et al., 2020).

\section{ASPECTOS ÉTICOS E SOCIAIS RELACIONADOS AO CUIDADO E ALOCAÇÃO DE RECURSOS}

Os idosos representam um papel importante na sociedade em muitas comunidades, especialmente em países de médio e alto desenvolvimento, onde são cabeças altamente ativas da família, participam de atividades de voluntariado e são membros dinâmicos no mercado de trabalho. Embora 
a maioria desfrute de excelente saúde emocional e mental, observa-se elevada incidência de distúrbios mentais e físicos, como doenças neurológicas, diabetes, perda auditiva, perda de visão, etc. Além disso, representam uma parcela da população suscetível a sofrer com desrespeito, abuso, abandono ou negligência de seus filhos ou outros membros da comunidade (BUHEJI et al., 2020).

A proteção do idoso, enquanto grupo vulnerável, requer medidas especiais, que contemplem o isolamento social e a minimização da exposição à infecção, seja limitando a visita de familiares ou cuidadores no domicílio ou durante eventual hospitalização por outras doenças. Cumprir as medidas de controle à propagação do vírus conforme sugerido pelas autoridades sanitárias é essencial para todos. Como é grande a probabilidade de se estender esses cuidados por tempo indeterminado, a implementação de medidas de proteção às pessoas vulneráveis, a longo prazo, é imprescindível (KAR, 2020).

O rápido aumento da demanda de saúde devido ao COVID-19 exige que as equipes de saúde tomem decisões técnicas e éticas difíceis sobre o tratamento de idosos, modelos de atendimento e sistemas de triagem. Algoritmos e sistemas de pontuação estão sendo desenvolvidos para prever riscos de mortalidade em relação aos recursos mais limitados, como ventilação mecânica. O rastreamento do estado de fragilidade clínica está sendo proposto como uma ferramenta fundamental para auxiliar nesse processo de triagem (HUBBARD et al., 2020). Contudo, a idade por si só não deve orientar as decisões de alocação de recursos de saúde durante a pandemia de COVID-19. As decisões sobre a alocação de recursos de saúde devem levar em consideração as preferências do paciente e seus objetivos de tratamento, bem como fatores do paciente, como a pontuação da Escala de Fragilidade Clínica com base em seu estado duas semanas antes do início dos sintomas (MONTERO-ODASSO et al., 2020).

Pesquisa de Thyrian e colaboradores, na Alemanha (2020), determinou que durante os primeiros meses da pandemia, os pacientes idosos visitaram menos ou foram menos visitados pelas equipes médicas e de outros profissionais de serviços de saúde. Além disso, esses pacientes avaliaram a prestação de serviços ambulatoriais, clínicas-dia e terapias prescritas como piores do que antes da pandemia. Esses resultados reforçam a necessidade de dispender maior atenção à adequação e qualificação dos serviços de saúde em função das consequências significativas a longo prazo.

Em relação à renda, a população idosa também é afetada pela recessão econômica, mesmo que de maneiras diferentes. Em países ricos, o trabalhador idoso costuma exercer suas atividades em cargos de consultoria, não exigindo deslocamento e permitindo o trabalho remoto. Além disso, o salário desse trabalhador costuma complementar uma renda pré-existente, com impactos menos significativos na economia doméstica. Nesta faixa etária, a probabilidade de deixar a força de trabalho voluntariamente é maior (BUI; BUTTON; PICCIOTTI, 2020). Já em países pobres e em desenvolvimento, o idoso representa muitas vezes a única fonte de renda, possuindo a responsabilidade financeira da família, apesar do senso comum em estimá-los como aposentados e pensionistas (HAMMERSCHMIDT; SANTANA, 2020). 
PREVENÇÃO, REABILITAÇÃO E PALIAÇÃO DE COVID-19 EM IDOSOS

Sem dúvida, pacientes que já possuem co-morbidades são mais suscetíveis ao adoecimento por COVID-19. Vaitheswaran e colaboradores (2020) investigaram a percepção de cuidadores de pessoas idosas com demência frente à doença e demonstraram que grande preocupação com a manutenção da higienização das mãos, uso de máscara facial e normas de distanciamento social pelas pessoas com deficiência. Outra importante preocupação está relacionada à saúde do cuidador, que precisa lidar com as mudanças nas rotinas diárias, elevado nível de atividades e intensificação de conexões sociais com as pessoas com demências desde o início da pandemia. Muitos pacientes não conseguem ir ao ar livre e realizar atividades que costumavam mantê-las ocupadas, e por isso, os cuidadores demonstram preocupação em não poder envolver seus familiares com demência em atividades dentro de casa.

A prevenção da exacerbação de comorbidades tem preocupado profissionais de saúde, que recomendam a prática de atividade física e exercícios para manter um estado de saúde adequado, neutralizar as consequências negativas de certas doenças como depressão, ansiedade, diabetes, hipertensão, doenças cardiovasculares, doenças respiratórias ou mesmo simplesmente para garantir um envelhecimento ativo, reduzindo o risco de fragilidade, sarcopenia e demência, como doenças associadas em pessoas idosas. Já os fatores estressores incluem maior duração da quarentena, medo de infecção, frustração, tédio, suprimentos inadequados, informações incompletas, perda financeira e estigma (JIMÉNEZ-PAVÓN; CARBONELL-BAEZA; LAVIE, 2020).

As estratégias de reabilitação do paciente idoso precisam abordar não apenas a ampla gama de déficits causados pela doença COVID-19, mas também devem ser capazes de fornecer reabilitação às pessoas com múltiplas fragilidades e doenças pré-existentes. Espera-se que as questões sociais mais amplas, como solidão, luto e pobreza, tenham um impacto maior na qualidade de vida dos idosos. Combinados, esses fatores condicionantes podem piorar a saúde e as funções físicas e psicológicas de idosos, que se refletem em síndromes de fragilidade (por exemplo, quedas e sarcopenia), déficits cardiorrespiratórios e neurológicos para os quais muitos serviços para idosos são configurados (DE BIASE et al., 2020).

Para o paciente idoso em cuidados paliativos, diversas medidas farmacológicas são indicadas, tais como o manejo da febre, da dificuldade e do desconforto respiratório, da ansiedade, da náusea, da dor, do delírio e da xerostomia. Como a condição do paciente pode piorar muito rapidamente, as prescrições farmacológicas devem ser feitas com antecedência para os problemas que podem surgir e documentadas em um plano de emergência. Os medicamentos, juntamente com os equipamentos necessários à sua administração, devem estar disponíveis no local do atendimento. Deve-se dar preferência às formas de dosagem oral, mas formulações subcutâneas alternativas também devem ser prescritas (KUNZ; MINDER, 2020). 
Durante esta crise e isolamento, cuidar das necessidades básicas dos idosos, como alimentação e nutrição, bem como continuar a cuidar de suas doenças pré-existentes, configura-se em uma estratégia de apoio fundamental. Deve-se considerar que existem necessidades distintas para idosos de áreas rurais e urbanas em função do acesso ao alimento de qualidade, o que implica na necessidade de desenvolvimento e implementação de políticas públicas que garantam uma alimentação adequada também no período da pandemia (KAR, 2020). A prática do isolamento social também pode restringir o acesso a alimentos saudáveis e nutritivos aos idosos com renda menor, levando à desnutrição e ao enfraquecimento do sistema imunológico, aumentando o risco de morbimortalidade por COVID-19 (BUHEJI et al., 2020).

Em relação à qualidade nutricional dos alimentos, é preciso destacar que dietas ricas em ácidos graxos saturados (SFAs), comuns no ocidente, podem levar à ativação crônica do sistema imunológico inato e à inibição do sistema imunológico adaptativo. Dessa forma, ocorre a indução de um estado lipotóxico e a ativação do sistema imune inato, por meio de macrófagos, células dendríticas e neutrófilos. Isso desencadeia a ativação de vias de sinalização inflamatórias, que por sua vez produzem mediadores pró-inflamatórios e outros efetores do sistema imunológico inato, que estão envolvidas na inflamação do tecido pulmonar e dano alveolar na patologia por COVID-19 (BUTLER; BARRIENTOS, 2020).

\section{PESQUISAS PARA O TRATAMENTO FARMACOLÓGICO DA COVID-19 EM IDOSOS}

O uso de medicamentos em idosos é complexo devido a uma variedade de fatores: fragilidade, representação limitada de pesquisas e alterações fisiológicas. Fragilidade refere-se a pessoas idosas que possuem reserva fisiológica reduzida e maior sensibilidade ao agente estressor. Ambas as características-chave contribuem para a vulnerabilidade dessa população aos medicamentos. Os ensaios clínicos de medicamentos têm restrições de idade e geralmente limitam a porcentagem de pacientes idosos inscritos (PAGE et al., 2016).

O acompanhamento farmacoterapêutico e o uso racional de medicamentos por pacientes idosos tem encontrado dificuldades em tempos de pandemia, pela redução do acesso aos serviços de saúde e aumento da automedicação. Como as interações pessoais não essenciais estão em espera, a saúde se adaptou para fornecer esses serviços eletronicamente por meio de videoconferência e telefone. A videoconferência pode imitar melhor a experiência pessoal, permitindo que os profissionais de saúde realizem algumas de suas avaliações de maneira visual (ELBEDDINI et al., 2020). No Brasil, a prescrição digital também representou um significativo avanço para melhoria do acesso à assistência farmacêutica (CRF-RS, 2020). 
As evidências para o uso de hidroxicloroquina como um fármaco de escolha para o tratamento de COVID-19 foi perdendo credibilidade à medida que os estudos randomizados apontaram falta de eficácia e problemas de segurança (SINGH et al., 2020; BOULWARE et al., 2020). Outros 48 compostos relacionados à cloroquina, além da azitromicina e doxicilina também foram propostos por ter um possível benefício terapêutico no tratamento da COVID-19, especialmente em idosos, mas ainda necessitam de maiores investigações (TRIPATHY, 2020).

\section{CONCLUSÃO}

Este artigo apresenta uma breve revisão sobre o impacto da pandemia por COVID-19 em pacientes idosos, bem como descreve estratégias de manejo dos problemas encontrados. Em meio a um cenário incerto, em que a pandemia exibe rápidas mudanças em termos de evidências, conhecimentos e as diretrizes, existe uma lacuna quando se trata da população que está sendo mais fortemente atingida, ou seja, o paciente idoso.

Destaca-se a necessidade de manter o suporte emocional, social, financeiro e de saúde para os idosos durante o período de pandemia. O acompanhamento próximo desse grupo populacional permitirá observar precocemente as manifestações clínicas da doença e realizar o acompanhamento da evolução do quadro. O mesmo pode-se inferir a respeito da saúde mental dos idosos, que apesar do isolamento social, devem se sentir acolhidos pelas suas famílias e pela sociedade.

Reforça-se a importância da revisão contínua dos resultados de pesquisas publicados neste momento, em função da impossibilidade de consolidação das evidências, o que pode implicar em contínuas modificações nas recomendações e condutas para o enfrentamento da doença. Ë de grande relevância a maciça produção de artigos como referência para auxiliar pesquisadores e profissionais de saúde a ter uma ideia objetiva do que está sendo feito e onde ainda é necessário avançar no cuidado ao idoso em uma pandemia.

\section{REFERÊNCIAS}

ARMITAGE, Richard; NELLUMS, Laura B. COVID-19 and the consequences of isolating the elderly. The Lancet Public Health, v. 5, n. 5, p. e256, 2020.

BOULWARE, David R. et al. A randomized trial of hydroxychloroquine as postexposure prophylaxis for Covid-19. New England Journal of Medicine, 2020.

BUHEJI, Mohamed et al. Mitigation of Risks of Complications and Deaths among the Elderly during Pandemics. American Journal of Medicine and Medical Sciences, n. 10, v. 7, p. 494-502, 2020. 
BUI, Truc Thi Mai; BUTTON, Patrick; PICCIOTTI, Elyce G. Early Evidence on the Impact of COVID-19 and the Recession on Older Workers. National Bureau of Economic Research, 2020.

BUTLER, Michael J.; BARRIENTOS, Ruth M. The impact of nutrition on COVID-19 susceptibility and long-term consequences. Brain, Behavior, and Immunity, 2020.

CRF-RS. Conselho Regional de Farmácia do Rio Grande do Sul. Saiba tudo sobre a prescrição digital. Disponível em: https://bit.ly/3nGENKq. Acesso em 20/08/2020.

DE BIASE, Sarah et al. The COVID-19 rehabilitation pandemic. Age and Ageing, p. 1-5, 2020.

DHAR, Debojyoti; MOHANTY, Abhishek. Gut microbiota and Covid-19-possible link and implications. Virus Research, p. 198018, 2020.

ELBEDDINI, Ali et al. Barriers to conducting deprescribing in the elderly population amid the COVID-19 pandemic. Research in Social and Administrative Pharmacy, 2020.

GIRDHAR, Ritika; SRIVASTAVA, Vivek; SETHI, Sujata. Managing mental health issues among elderly during COVID-19 pandemic. Journal of Geriatric Care and Research, v. 7, n. 1, 2020.

HAMMERSCHMIDT, Karina Silveira de Almeida; SANTANA, Rosimere Ferreira. Saúde do idoso em tempos de pandemia COVID-19. Cogitare Enfermagem, v. 25, 2020.

HUBBARD, Ruth E. et al. Frailty in the Face of COVID-19. Age and ageing, 2020.

JIMÉNEZ-PAVÓN, David; CARBONELL-BAEZA, Ana; LAVIE, Carl J. Physical exercise as therapy to fight against the mental and physical consequences of COVID-19 quarantine: Special focus in older people. Progress in cardiovascular diseases, 2020.

KÄLL, A., JÄGHOLM, S., HESSER, H., ANDERSSON, F., MATHALDI, A., NORKVIST, B. T., ANDERSSON, G. (2020). Internet-based cognitive behavior therapy for loneliness: a pilot randomized controlled trial. Behavior therapy, n. 51, v. 1, 54-68, 2020.

KAR, N. Covid-19 and older adults: in the face of a global disaster. Journal of Geriatric Care and Research, v. 7, n. 1, p. 1-2, 2020. 
KUNZ, Roland; MINDER, Markus. COVID-19 pandemic: palliative care for elderly and frail patients at home and in residential and nursing homes. Swiss Medical Weekly, v. 150, n. 1314, 2020.

MONTERO-ODASSO, Manuel et al. Age alone is not adequate to determine healthcare resource allocation during the COVID-19 pandemic. Canadian Geriatrics Journal, v. 23, n. 1, p. 152-154, 2020.

MUELLER, Amber L.; MCNAMARA, Maeve S.; SINCLAIR, David A. Why does COVID-19 disproportionately affect the elderly?. Aging. v. 12, n. 10, p. 9959-9981, 2020.

NEWMAN, Michelle G.; NUR, Hani Zainal. The value of maintaining social connections for mental health in older people. The Lancet Public Health n. 5, v. 1, e12-e13, 2020.

OMURA, Takuya et al. Geriatric practice during and after the COVID-19 pandemic. Geriatrics \& Gerontology International, [s.v. ], [s.n], [s.p. ], 2020.

PAGE, Amy Theresa et al. Deprescribing in older people. Maturitas, v. 91, p. 115-134, 2016.

SINGH, Awadhesh Kumar et al. Chloroquine and hydroxychloroquine in the treatment of COVID-19 with or without diabetes: A systematic search and a narrative review with a special reference to India and other developing countries. Diabetes \& Metabolic Syndrome: Clinical Research \& Reviews, 2020.

STEPTOE, Andrew et al. Social isolation, loneliness, and all-cause mortality in older men and women. Proceedings of the National Academy of Sciences, v. 110, n. 15, p. 5797-5801, 2013.

THYRIAN, Jochen René et al. The situation of elderly with cognitive impairment living at home during lockdown in the Corona-pandemic in Germany. Research Square, [s.n], [s.v. ], p. 1-25, 2020.

TRIPATHY, Swagata. The COVID-19 pandemic and the elderly patient: review of current literature and knowledgebase. Journal of Geriatric Care and Research, v. 7, n. 2, 2020.

VAITHESWARAN, Sridhar et al. Experiences and needs of caregivers of persons with dementia in India during the COVID-19 pandemic-A Qualitative Study. The American Journal of Geriatric Psychiatry, 2020.

WAND, Anne Pamela Frances et al. Covid-19: The implications for suicide in older adults. International Psychogeriatrics, p. 1-16, 2020. 